INVESTIGACIÓN/RESEARCH

\title{
LA PIZARRA DIGITAL INTERACTIVA COMO RECURSO POTENCIADOR DE LA MOTIVACIÓN
}

Celia González Carrasco: Universidad de Castilla La Mancha. España.

Celia.Gonzalez2@alu.uclm.es

José Francisco Durán Medina2: Universidad de Castilla La Mancha. España. JoseFrancisco.Duran@uclm.es

\section{RESUMEN}

La motivación del estudiante es un factor clave en su aprendizaje. No obstante, cualquier docente habrá observado cómo un alto porcentaje de alumnos pierde motivación conforme avanza desde los cursos de Educación Infantil a Educación Primaria, llegando muchos de ellos a bajar su rendimiento escolar. Así, en Educación Infantil se puede observar la gran motivación de los alumnos cuando comienzan su trayectoria educativa, mostrando interés por todo y participando en cualquier actividad propuesta. Sin embargo, no sucede de igual manera en Educación Primaria, sobre todo en los últimos cursos de esta etapa. Tras estas claras diferencias en el cambio de actitud de los alumnos de Educación Primaria, surge el objetivo de este proyecto que es incrementar la motivación de éstos a través de la Pizarra Digital Interactiva, demostrando lo que dicen algunos autores: "La utilización de la Pizarra Digital en las aulas motiva a alumnos y profesores" (Gallego y Dulac, 2005). Probablemente no será tarea fácil, pero será un avance en este novedoso recurso. A tal fin se dirigió este proyecto, con el propósito de averiguar si la utilización en entornos educativos de la Pizarra Digital Interactiva pudiera conseguir ese aumento de motivación. Mediante una serie de cuestionarios oportunamente validados, se obtuvieron numerosas respuestas por parte de docentes y discentes, cuyo análisis vino a determinar, entre otras cosas, la veracidad del título de nuestro artículo.

\section{PALABRAS CLAVE}

Pizarra Digital Interactiva - Motivación - Educación - Educación primaria - TIC Hardware - Magisterio - Investigación - Recursos

\footnotetext{
${ }^{1}$ Celia González Carrasco: Egresada en Magisterio con la especialidad de Primaria en la Facultad de Educación de Toledo.

Celia.Gonzalez2@alu.uclm.es

${ }^{1}$ José Francisco Durán Medina: Profesor Doctor en la Facultad de Educación de Toledo. Mención TICE (Tecnologías de la Información y de la Comunicación en Educación).

JoseFrancisco.Duran@uclm.es
} 


\title{
THE INTERACTIVE WHITEBOARD AS AN ENHANCER RESOURCE OF MOTIVATION
}

\begin{abstract}
Student motivation is a key factor in their learning. However, any teacher will have noticed how a high percentage of students lose motivation as they progress from Preschool Education to Elementary Education, many of them dropping their school performance. This way, in Pre-school Education the great motivation of students can be noticed when they start their study, showing interest everything and participating in any activity. However it doesn't happen the same in Elementary Education, above all the last years of this stage. After these clear differences in attitude change of the students of Elementary Education the objective of this project arises: to increase student motivation through interactive whiteboard, proving what some authors say: "The use of the whiteboard in the classroom motivates students and teachers" (Gallego y Dulac, 2005). Probably it will not be easy task, but it will be an advance in this new resource. To this end the project walked, in order to find out if the use of the interactive whiteboard in educational enviroments could get that increase motivation. Through a series of timely validated questionnaires, many responses were obtained by teachers and students, whose analysis came to determine, among other things, the accuracy of the title of our article.
\end{abstract}

\section{KEY WORDS}

Interactive whiteboard - Motivation - Education - Primary education - ICT Hardware - Teaching - Research - Resources.

\section{INTRODUCCIÓN}

En los últimos años, son muchos los jóvenes campeones que han situado a España en la cima del éxito deportivo. Sin embargo, no puede decirse lo mismo en la educación obligatoria, donde también somos triunfadores, campeones del fracaso escolar. Así, según los datos recogidos por la Unesco en la edición 2012 del estudio anual Educación para todos, uno de cada tres jóvenes españoles de entre 15 y 24 años dejó sus estudios antes de acabar la enseñanza secundaria. Ante esta escalofriante cifra surge el gran interrogante tratado por diferentes autores, ¿cuáles son sus causas?: "La desmotivación explica el fracaso escolar" (Puig, 2006). "Si hay desmotivación escolar, siempre ocurrirá posteriormente el fracaso" (Bustos, 2001). Podría seguir escribiendo citas textuales similares. Y es que realmente, la motivación es uno de los factores que influyen en el fracaso escolar.

Pero, ¿existe una estrategia didáctica para enriquecer el proceso de enseñanzaaprendizaje de motivación? Por desgracia no, pero sin embargo en los últimos años, con la aparición de las Tecnologías de la Información y la Comunicación en el ámbito 
educativo, diferentes autores han expuesto el "resucitar" de la motivación gracias al uso de algunas de esas herramientas, una de ellas la Pizarra Digital Interactiva: “La pizarra digital interactiva permite una progresiva innovación en las prácticas docentes" (Miller y Glover, 2002), "una mejora de la motivación y atención de los alumnos" (Beeland, 2002) "y la disponibilidad de nuevas herramientas para atender la diversidad de los alumnos, especialmente a aquellos alumnos con discapacidades o dificultades severas o moderadas para el aprendizaje" (Pugh, 2001). "Los alumnos están más atentos, motivados e interesados por las asignaturas incluso en aquellos entornos en los que les resulta difícil mantener la disciplina. Los estudiantes se sienten como en casa, ante el mundo audiovisual del televisor, concentrados con sus videojuegos o navegando lúdicamente por Internet. Los temas que se tratan en clase se aproximan más a sus experiencias previas. Les resulta más fácil relacionar lo nuevo con lo que ya saben. Pueden realizar unos aprendizajes más significativos" (Marqués et al., 2006). "Aumenta la satisfacción y la motivación tanto en los docentes como en los discentes, gracias al uso de fuentes más variadas, dinámicas y divertidas" (Levy, 2002).

Como podemos ver, la Pizarra Digital Interactiva en la actualidad es el recurso tecnoeducativo con más fuerza en el contexto de la educación y la formación en el siglo XXI. Es frecuente encontrarnos en diferentes medios de comunicación e información la innovación educativa que este recurso supone, así como las inversiones realizadas o planificadas por las diferentes administraciones educativas en ello. Pero, ¿realmente el uso de la Pizarra Digital Interactiva incrementa la motivación de los alumnos? Es un interrogante que diferentes autores ya han afirmado, no obstante, a través de este proyecto se ha investigado.

Para ello, se ha considerado necesario sustentar esta investigación con un marco teórico antes de comenzar con la aplicación práctica, por lo que fue necesario adentrarse en diferentes trabajos e investigaciones que abordasen los dos factores que lo ocupaban: la motivación y la Pizarra Digital Interactiva. Terminada la primera etapa de la investigación, el trabajo se dirigió al núcleo de este trabajo, la recogida de los datos empíricos y sus respectivas conclusiones.

\subsection{TEORÍAS PREVIAS Y ESTADO DE LA CUESTIÓN}

\subsection{Teorías sobre la motivación.}

La motivación ha sido, es y será un tema ampliamente estudiado en todos los niveles, entre ellos el educativo donde es considerada un factor importante en el aprendizaje de los niños, así Robert E. Slavin señala que "los estudiantes que no están motivados no aprenden" (Slavin, 1987). Tanto es así que, existen multitud de teorías acerca de ello, no obstante, para mantener la extensión de este estudio dentro de límites razonables se expondrá un breve resumen de las teorías más importantes basado en las ideas expuestas por K.B. Madsen en Teorías de la motivación (Madsen, 1972).

En las teorías de la motivación de la antigüedad, Aristóteles y otros filósofos describieron el deseo o los impulsos como una de las fuerzas mentales o facultades ubicadas en el mismo nivel de la percepción, la imaginación y el sentimiento. En la Edad Media, Tomás de Aquino y otros distinguieron el deseo sensual de la voluntad 
racional. Para Descartes, Hobbes y Spinoza, filósofos de una época más moderna, los impulsos eran todavía una clase importante de variables psicológicas, de importancia equiparable a la de los procesos afectivos e intelectuales. Durante los siglos XVIII y XIX, el hedonismo entendía que el hombre siempre actúa para lograr placer o evitar el desplacer. Por su parte los empiristas ingleses Locke, Berkeley y Hume consideraban que las variables intelectuales prevalecían considerablemente sobre los otros tipos de variables psicológicas. Con la aportación de Kant a la psicología la cognición, la emoción y la voluntad se pusieron en el mismo nivel de clasificación psicológica, tripartición que prevaleció hasta principios de siglo. W. Wundt definió la voluntad como una serie especial de emociones que concluye con un sentimiento determinista que espontáneamente se traduce en acción. A finales del siglo XIX, influidos por la teoría de Darwin, los psicólogos comenzaron a ver en los instintos los motivos primarios de la conducta, tanto en el hombre como en los animales; así, W. James, sostuvo que el hombre era la criatura que poseía más instintos y que éstos estaban en relación primaria con la voluntad.

Más tarde, en el siglo XX también se desarrollaron distintas teorías acerca de la motivación. Así los instintos dominaron en la teoría de McDougall (1871-1938), psicólogo que destacó con energía la importancia de la motivación, pues según su teoría "todos los procesos vitales (incluso la vida mental y la conducta) son intencionistas" (McDougall, 1932) y están motivados por propensiones innatas.

Edward Chase Tolman (1886-1959), fue uno de los autores que mayor influencia ejerció sobre el desarrollo de la psicología teórica, llegando a formular una cantidad considerable de conceptos teórico-psicológicos: variable interviniente, conductismo moral, etc. (Tolman, 1932). Su teoría motivacional expone que toda conducta es determinada por varias variables empíricas e hipotéticas cooperantes, y motivada por impulsos, que son condiciones biológicas de desequilibrio.

Por su parte, Paul Thomas Young en su libro Motivation of Behavior (1936) formuló su teoría de la motivación que resumida en un axioma motivacional expone que toda conducta está motivada por una liberación y regulación de la energía causada por estímulos internos o externos.

El psicólogo norteamericano Gordon W. Allport (1897-1967) realizó una importante contribución a la psicología general con su definición de la personalidad y sus hipótesis sobre la autonomía funcional en los individuos adultos con su libro A psychological interpretation (1937). Su teoría motivacional declara que la conducta es determinada por variables psicológicas dinámicas (rasgos motivacionales) que en los adultos pueden funcionar independientemente de las necesidades biológicas, pero que entonces son influidos por estímulos externos.

Por otro lado, Kurt Lewin (1890-1947) de acuerdo con su libro Behavior and Develepmont as a Function of the Total Situation (1946) presentó su teoría según la cual la conducta (C) de un individuo será siempre función (F) de la situación total, el espacio vital (Ev), que incluye las condiciones del individuo $(\mathrm{P})$ y las del ambiente (A), factores éstos estrechamente interdependientes, por tanto para predecir la conducta de un individuo es entonces necesario conocer el espacio vital en un momento dado, y las hipótesis que formulan las relaciones funcionales entre el espacio vital y la conducta. 
Una teoría muy interesante y fructífera de la motivación es la del psicólogo norteamericano Henry A. Murray (1893-1988) según la cual toda conducta es motivada por necesidades que son procesos cerebrales dominantes determinados por procesos fisiológicos o por situaciones-presión, que pueden influir sobre las condiciones bio-sociales de la vida del individuo.

Respecto a la teoría de la motivación de Clark L. Hurk (1884-1952) muestra que ni la más simple reacción innata puede existir sin motivación, la cual es en consecuencia una condición necesaria de la aparición de la conducta.

En referencia a la teoría de Tinbergen (1907-1988) es una de las más valiosas teorías psicológicas modernas, porque constituye un eslabón entre la psicología y el resto de la biología, así esta teoría formula que las respuestas instintivas están determinadas por factores externos que determinan principalmente la iniciación y orientación de la conducta e internos que determinan la motivación del sistema nervioso central y la conducta.

El psicólogo David C. McClelland (1917-1998) ha contribuido a la psicología de la motivación con una serie de artículos experimentales y teóricos. En su libro Studies in Motivation (1955) define el motivo como una asociación afectiva, que se manifiesta como conducta intencionista determinada por la asociación previa de señales y el placer o dolor (McClelland, 1955). Según esta definición, todos los motivos son adquiridos, y toda motivación se basa en emociones; distinguiéndose entre dos tipos de motivos: el positivo o de aproximación, que es una expectativa de placer o satisfacción, y el negativo o de evitación, que es una expectativa de desplacer o dolor. También se ha considerado conveniente nombrar, aunque sea de manera muy abreviada, otras teorías existentes representativas de este período como son la teoría de Frenkel-Brunswik (1942) que define el impulso como la variable más importante y la única motivacional; la teoría de Masserman (1946) que en su primer principio biodinámico denominado "principio de la motivación" define a la necesidad como la variable motivacional más importante, entendida como una deficiencia o desequilibrio fisiológico trasladado dinámicamente a la conducta; la teoría de Freeman, en su Capítulo V señala que "toda conducta total está motivada, por lo menos en el sentido de disparar algunos desplazamientos orgánicos" (Freeman, 1948); la teoría de Moore (1948) que en su libro The Driving Forces of Human Nature (1948) define al deseo como un "anhelo que experimentamos de buscar o producir una situación en la que sean satisfechas las tendencias impulsivas y los deseos naturales" (Moore, 1948) ; por su parte, el psicólogo norteamericano Maier define la motivación como el "proceso que determina la manifestación de la conducta, o influye sobre su expresión futura, por las consecuencias a que tal conducta conduce" (Maier, 1949) ; la teoría de Cattell (1950) expone que toda acción expresa una cooperación entre los rasgos de temperamento, de habilidad y dinámicos, o está determinada por ella, pero en grados variables; por su parte en la teoría de Thomas M. French (1952) la necesidad y la esperanza son consideradas las variables motivacionales más importantes; en el comienzo introductorio de la teoría de Ross Stagner y T. F. Karwoski (1952) se señala la importancia de la motivación afirmándose que la "motivación proporciona la potencia que impele a la conducta adaptativa, incluso al aprendizaje, a la comprensión del medio, al pensamiento y al razonamiento" (Stagner y Karwoski, 1952); la teoría de Skinner (1953) expone que las 
variables que pueden explicar la conducta de un individuo no están en el interior del organismo, sino en su ambiente inmediato, en su historia ambiental; por último la teoría del psicólogo danés Holt-Hansen (1956) afirma que la motivación viene dada por las necesidades del individuo.

Por último, para finalizar este apartado, cabe mencionar algunos estudios realizados en los últimos años sobre la relación motivación-Tecnologías de la Información y la Comunicación en educación. Según estas investigaciones, el uso de las TIC en las aulas provoca en los alumnos un aumento de su interés y motivación; así diferentes especialistas e investigadores en este campo ya lo han ratificado como Manuel Area (2010), María Domingo y Pere Marquès (2011) u otros como Micaela Manso, Paula Pérez, Marta Libedinsky, Daniel Light y Magdalena Garzón (2011).

\subsection{Teorías sobre la Pizarra Digital Interactiva.}

En los últimos años, la implantación de las Tecnologías de la Información y la Comunicación en todos los ámbitos de la sociedad es un hecho incuestionable, pero su nivel de integración en las aulas es todavía un proceso que muchos especialistas han tipificado como arduo y lleno de obstáculos. Así, a finales de los ochenta y principios de los noventa el ordenador fue difundido por las escuelas. A mediados de los noventa, la difusión de Internet promovió el aprendizaje a través de textos e imágenes. Un poco más tarde, a finales de los noventa y en los albores del siglo XXI se introdujo una nueva metodología conocida con el nombre de e-learning. Y durante la primera década del presente siglo, se han hecho populares algunas herramientas innovadoras entre la que podemos mencionar la denominada Pizarra Digital Interactiva.

Con la integración de estas tecnologías en el ámbito educativo, los roles del profesor y el alumno se han visto renovados, de modo que el profesor ya no es el instructor de los contenidos sino un asesor, orientador, facilitador y mediador del proceso de enseñanza-aprendizaje. Por su parte, el alumno no es un acumulador de conocimientos, sino que sobre todo debe saber usarlos, para lo que precisa aprender a buscar información, evaluarla y convertirla en conocimiento, es decir, debe aprender a aprender.

Los niños tienen la necesidad de conocer y hacer uso de las TIC y así lo demuestra un dato del Instituto Nacional de Estadística del año 2011, pues 2.572.073 niños entre 10 y 15 años hacen uso de las Tecnologías de la Información y Comunicación en sus hogares. Con tal dato significativo, en el Real Decreto 1513/2006, del 7 de Diciembre por el que se establecen las enseñanzas mínimas de la Educación Primaria, se recoge que el tratamiento de la información y competencia digital es una de las competencias básicas que el alumnado debe adquirir en la enseñanza básica. Esta competencia recoge que el alumno ha de ser competente para buscar, localizar, organizar y comunicar información utilizando los elementos básicos del ordenador y las utilidades que éste tiene (navegar por Internet, comunicarse por correo electrónico, utilizar procesadores de texto...). Por este motivo, en el año 2009 se pone en marcha el proyecto Escuela 2.0 por parte del Ministerio de Educación y de las Comunidades Autónomas que colaboran, para innovar y modernizar los sistemas de enseñanza con la integración de las TIC tanto en Educación Primaria como en 
Educación Secundaria Obligatoria. Con este proyecto, una de las herramientas educativas que se ha introducido en el aula es la Pizarra Digital Interactiva.

Para dar una definición exacta de este recurso, en primer lugar es necesario hacer una distinción y es que no todas las pizarras son digitales e interactivas. Una pizarra digital es un "sistema tecnológico integrado por un ordenador multimedia conectado a Internet y un videoproyector que presenta sobre una pantalla o pared de gran tamaño lo que muestra el monitor del ordenador" (Marquès y Grupo DIM, 2006). La Pizarra Digital Interactiva por su parte, se diferencia de la anterior porque añade un dispositivo de control de puntero que permite la interacción directa sobre la superficie de proyección de contenidos digitales, en un formato idóneo para visualización en grupo (Marquès, 2008). Existen varios modelos en el mercado y aunque la mayoría de ellos mantienen unas características comunes y básicas, se encuentran diferencias importantes que pueden condicionar su adecuación y conveniencia. Algunos de los tipos de Pizarras Digital Interactivas existentes actualmente en el mercado son: eBeam, Hitachi, Smartboard, TeamBoard, Mimio, Activboard, Promethean, Numonics, StarBoard y èno, entre otras. Pese a que su creación data de hace más de una década, su uso aún no se ha generalizado. En el ámbito internacional Reino Unido, Estados Unidos y Australia son los países con una mayor implantación de esta tecnología por la fuerte inversión que han desarrollado sus gobiernos (Armstrong, 2005; Holmes, 2009). Le siguen países como Canadá, Francia, Dinamarca y México, lugares donde también se han efectuado un importante número de investigaciones. Si bien esa adopción a gran escala es un fenómeno del Reino Unido, hay un interés creciente en el potencial de esta tecnología en todo el mundo (Bell, 2002; Hodge y Anderson, 2007). En el ámbito nacional, según el informe Red.es (2006) del Ministerio de Industria, Turismo y Comercio de España, el número de Pizarras Digitales Interactivas disponibles en centros de formación de profesores y en centros educativos de enseñanzas no universitarias rondaba las 700 unidades. Una información más reciente del profesor e investigador José Dulac, indica que en el año 2009 la introducción de las Pizarras Digitales Interactivas puede suponer un $12 \%$ y que los profesores que están capacitados para trabajar con esta tecnología están por debajo del 10\% (León, 2009).

Como se puede observar, la Pizarra Digital Interactiva es una herramienta que ha revolucionado el mundo y como ya preveían Domingo Gallego y Nibaldo Gatica en el año 2000 pronto se incorporaría al mundo educativo; sin embargo en ese mismo año, Greiffnhagen (2000) a pesar de señalar su potencial educativo, demanda la falta de estudios empíricos que lo demuestren. Será a partir de ese momento cuando comenzarán a realizarse diferentes estudios destinados a señalar cuáles son los efectos que se evidencian tras la incorporación de estas tecnologías en la educación. Algunos aspectos que se investigan son la motivación o la facilidad para la comprensión de los conceptos con el uso de las Pizarras Digitales Interactivas (Beeland, 2002; Miller y Glover, 2002). Otros giran en torno a las percepciones y actitudes de los alumnos con respecto a la PDI (Goodison, 2002; Hall y Higgings, 2005) o sobre su uso por parte de los profesores (Cogill, 2002; Kennewell y Morgan, 2003). También existen estudios de caso de su utilización concreta en diferentes contextos y niveles educativos (Lee y Boyle, 2003; Miller y Glover, 2002, 2006) e informes que evalúan el uso de la PDI (Smith, 2000). Finalmente, hay publicaciones 
que se limitan a realizar una revisión de la literatura existente hasta el momento (SMART, 2005; Smith et al., 2005).

El uso de la Pizarra Digital Interactiva resulta sencillo para la mayoría de las personas ya que requiere de conocimientos informáticos básicos. Aunque Quashie (2009) atribuye la responsabilidad de su manejo a los docentes, con el fin de desarrollar lecciones interactivas que atraigan y motiven a sus alumnos. Además, Gallego, D. y Dulac, J. (2005) consideran "necesaria la formación técnica, pedagógica, metodológica y que potencie la creatividad para la correcta utilización y aprovechamiento" de esta herramienta. En general, son múltiples las posibilidades y acciones que cada docente puede explotar en función de sus propios usos, necesidades y experiencias. San Pedro (2008) señala que la PDI muestra todo su potencial cuando se utiliza como una aplicación o como un entorno de aplicaciones, y en este caso como un escritorio virtual específico en el que se integran todas las aplicaciones contenidas en un ordenador. Además, tienen una gran ventaja y es que están adaptadas para todas las edades, desde preescolar hasta la educación superior (Smith et al., 2005).

Con el fin de identificar las buenas prácticas que los docentes hacen con el uso de la PDI, Haldane y Somekh (2005) describen un modelo de enseñanza docente de cinco niveles: nivel 1 de base o fundamental, el docente lo utiliza como herramienta de presentación; nivel 2 formativo, el maestro comienza a usar las funcionalidades más simples como el lápiz electrónico y el borrador; nivel 3 facilidad, el maestro domina todas las funcionalidades disponibles en la PDI y comienza a usarla con gran frecuencia y facilidad; nivel 4 fluidez, los docentes sienten que hay horizontes por explorar y se convierten en cazadores y buscadores activos y cosechan nuevas ideas y nuevos contenidos; nivel 5 vuelan, los maestros a este nivel son virtuosos ejecutantes de la PDI con un amplio repertorio, técnicas e interacción con los estudiantes. Además, Kennewell (2001) señala la importancia del uso de esta herramienta no solo por parte del maestro, sino por parte del alumno pues considera que es vital en el aumento de la motivación y del aprendizaje.

Por su parte, Jenny Gage (2002) expone que la PDI puede ser muy útil para las matemáticas como resultado de su capacidad para que el usuario pueda dibujar líneas rectas, círculos, triángulos y cuadrados; así un estudio sobre la enseñanza de las matemáticas de Clemens, Moore y Nelson (2001) llega a la conclusión de que importantes logros académicos y actitudes positivas en el alumnado son resultado del uso de la Pizarra Digital SmartBoard. Además, Forrest (2005) señala ser un elemento indispensable en sus clases de matemáticas.

Con respecto a los beneficios que este recurso ofrece en educación, Smith (1999) señala ser un recurso muy versátil, con aplicaciones para todas las edades y todas las áreas curriculares. Bryant y Hunton (2000) señalan la interacción entre el estudiante o profesor y el medio, aumentando de este modo la participación de los alumnos. Derek Glover y David Miller (2001) declaran que facilita a los profesores el compartir y utilizar varias veces materiales didácticos. Smith (2001) destaca una mayor comprensión de los conceptos más complejos por parte de los alumnos gracias a las presentaciones, más claras, más dinámicas y más eficientes. Por su parte, Beeland (2002) expone que fomenta tres tipos de aprendizaje: el visual. A través del uso de la PDI se puede abarcar desde el uso de textos y dibujos hasta la utilización de 
animaciones y vídeos; el auditivo, a través del uso de series de palabras para la pronunciación, discursos y poemas, además de la escucha de sonidos o música; y el táctil que permite a los estudiantes interactuar físicamente con la pizarra y puede ayudar a satisfacer sus necesidades.

El mismo autor concluye que la incorporación de estos tres tipos de aprendizaje en una unidad didáctica puede determinar la medida en que los estudiantes participan en el proceso de enseñanza-aprendizaje $\mathrm{y}$, por tanto, que están motivados para aprender. Goodison (2002) afirma que esta herramienta permite el acceso al ordenador sin utilizar el teclado, gracias a la macropantalla táctil, facilitando el uso de la informática a niños pequeños y a estudiantes de educación especial o con minusvalías. Bell (2002) declara ser un imán para los niños, además de poderse tener en cuenta los distintos estilos de aprendizaje de los alumnos ya que los profesores pueden acudir a muchas y variadas fuentes y recursos para responder a las necesidades específicas del alumno. Walker (2002) manifiesta otro de sus muchos beneficios: posibilita a los profesores el conservar e imprimir lo que está en la pizarra, incluyendo las notas realizadas durante la clase, facilitando la revisión.

Por su parte, Levy (2002) señala que aumenta la satisfacción y la motivación tanto en los docentes como en los discentes, gracias al uso de fuentes más variadas, dinámicas y divertidas y aumenta las oportunidades de participación y colaboración, ayudando a desarrollar en los alumnos las destrezas personales y sociales.

Edward, Hartnell y Martin (2002) exponen que no solo aumenta el disfrute de los alumnos en el aprendizaje, sino también por lo general da lugar a respuestas más precisas de los alumnos. Pere Marquès junto a Pilar Casals (2003) revelan que induce una notable renovación de las metodologías docentes y de los procesos de enseñanza aprendizaje, incrementa la motivación de los estudiantes, revitaliza la autoestima profesional de los profesores y facilita el logro de aprendizajes más significativos y acordes con la sociedad actual. Walker (2003) destaca que aumenta la disponibilidad de tiempo permitiendo al docente presentar con facilidad y eficacia recursos de internet o de otra fuente informática. Knight, Pennant y Piggott (2005) descubren que contribuye a aumentar la autoestima de algunos niños. Domingo Gallego y José Dulac (2005) aportan que la PDI mejora notablemente los procesos de enseñanzaaprendizaje, motiva a alumnos y profesores y además propicia la creatividad docente. José Dulac descubre que "la mayor motivación de profesores y alumnos usuarios de la Pizarra Digital lleva consigo una mejora de la autoestima y una participación más activa en la dinámica de la clase" (Dulac, 2006).

Investigadores como Higgins, Beauchamp y Miller afirman que podría ser "el cambio más significativo en el ambiente de aprendizaje del aula en la última década" (Higgins, Beauchamp y Miller, 2007). Martin (2007) en base a su investigación, concluye que es probable que este recurso aumente la motivación de los estudiantes y su participación. Nieto y Bode (2008) manifiestan ser apropiada para enseñar a los niños de diferentes orígenes culturales. Pere Marquès (2008) expone que aumenta la participación de los alumnos, aumenta la atención y retentiva de los estudiantes, motiva, aumenta la comprensión, facilita el tratamiento de la diversidad y ayuda en educación especial compensando problemas de visión, audición y psicomotricidad. En definitiva, podemos concluir que "la utilización de la Pizarra Digital en las aulas motiva a alumnos y profesores" (Gallego y Dulac, 2005). "Aumenta la satisfacción y 
la motivación tanto en los docentes como en los discentes, gracias al uso de fuentes más variadas, dinámicas y divertidas" (Levy, 2002).

\section{OBJETIVOS}

A partir de la revisión teórica y la intervención práctica en el ámbito educativo, se percibió un cambio de actitud en el alumnado con la integración de las TIC en el proceso de enseñanza-aprendizaje, siendo la principal protagonista la Pizarra Digital Interactiva. Y de ahí surgen los objetivos de este trabajo:

1. Conocer la integración de la Pizarra Digital Interactiva en las aulas de Tercer Ciclo de Educación Primaria.

2. Identificar los efectos de la Pizarra Digital Interactiva en el alumnado.

3. Constatar que la Pizarra Digital Interactiva provoca un cambio de actitud en el alumnado.

4. Comprobar que la Pizarra Digital Interactiva es un recurso potenciador de la motivación.

\section{METODOLOGÍA}

\subsection{Preguntas de Investigación.}

Concretamente, las cuestiones generales que han guiado la planificación y desarrollo del estudio fueron:

Para saber la integración de la PDI en el aula:

1. I1. ¿Hay una PDI en el aula?

2. I2. ¿El profesor utiliza la PDI?

3. I3. ¿Cuántos días a la semana se utiliza la PDI en el aula?

4. I4. ¿En qué asignaturas se usa la PDI?

5. I5. ¿Los alumnos manejan la PDI?

Para conocer si la utilización de la Pizarra Digital Interactiva potencia la motivación en los alumnos:

6. M1. ¿Aumenta el interés por las clases?

7. M2. ¿Incrementa la atención?

8. M3. ¿Anima a participar en las actividades propuestas?

9. M4. ¿Fomenta la comprensión de los contenidos abordados?

10. M5. ¿Favorece la variedad de actividades?

Con el fin de recopilar la información relativa al tema que aborda este trabajo se ha utilizado como instrumento de recogida de datos el cuestionario.

Éste nos permite realizar una doble interpretación cuantitativa y cualitativa de los datos. Es cuantitativa en la forma de recoger los datos puesto que se han recogido al final del proceso y se cuantifican para posibilitar su análisis. Pero al mismo tiempo es cualitativa porque los datos se interpretan no sólo a través del tratamiento estadístico, sino analizándolos y reagrupándolos por categorías que dan lugar a nuevos análisis y explicaciones. El procedimiento para la elaboración de un cuestionario es de primordial importancia para solventar cualquier problema y evitar 
errores insalvables posteriormente. En esta investigación se ha optado por dotar al cuestionario utilizado con la mayor rigurosidad, fiabilidad y validez posibles mediante la consulta a un grupo de personas a las que se supone un conocimiento elevado de la materia que se va a tratar. Los expertos colaboradores en esta investigación para confeccionar el cuestionario utilizado en la recogida de datos han sido (por orden alfabético):

1. Cruz Cruz, María Purificación. Profesora de Didáctica y Organización Escolar en la Universidad de Castilla La-Mancha.

2. Díaz Castromil, Javier. Profesor de ELE en Instituto Cervantes de Bucarest. Profesor de español para extranjeros desde mayo de 2004 y ha trabajado en diversos países Italia, Francia, Serbia y España tanto en instituciones públicas como en privadas.

3. Durán Medina, José Francisco. Profesor de Didáctica y Organización Escolar y coordinador del Máster de Secundaria en la Universidad de Castilla LaMancha. Investigador en varios Proyectos: "Ciudadanía Digital y Open Data Access: Empoderamiento ciudadano y medios sociales en el entorno digital" y "Las políticas de un ordenador por niño en España. Visiones y prácticas del profesorado ante el programa Escuela 2.0. Un análisis comparado entre comunidades autónomas".

4. Fernández Muñoz, Ricardo. Profesor de Didáctica y Organización Escolar en la Universidad de Castilla La-Mancha. Coordinador en la Universidad de Castilla- la Mancha de la Investigación: "Las políticas de un ordenador por niño en España. Visiones y prácticas del profesorado ante el programa Escuela 2.0. Un análisis comparado entre comunidades autónomas". Miembro del proyecto de innovación docente "AulaCiencia, wiki como espacio de trabajo común de las Facultades de Educación de la Universidad de Castilla-La Mancha junto con la Facultad de Educación de la Pontificia Universidad Católica de Chile, en el área de la didáctica de las ciencias experimentales.".

5. Macías Alegre, Adrián. Editor del Blog de Software Libre (COBDC) en Grup de Treball de Programari Lliure pels Professionals de la Informació. Miembro del Comité Científico de las XVII Jornadas Bibliotecarias de Andalucía en Asociación Andaluza de Bibliotecarios. Bibliotecario en Universidad Pablo de Olavide. Director Gerente en Dokumentalistas y Profesor en A4Formacion.

6. Martín Espinosa, Álvaro. Profesor de Teoría e Historia de la Educación en la Universidad de Castilla La-Mancha. Investigador en varios proyectos: "La escuela en la II República y en el Régimen Franquista. Historias de vida", investigador colaborador del Proyecto "Las políticas de un ordenador por niño en España. Visiones y prácticas del profesorado ante el programa ESCUELA 2.0" e investigador del Grupo Ciberimaginario-UCLM.

\subsection{Muestreo}


A efectos de nuestra investigación, la población queda definida por el profesorado y alumnado de Tercer Ciclo de Educación Primaria del Colegio de Educación Infantil y Primaria “Ángel del Alcázar" durante el curso 2012/2013.

La imposibilidad de que todo el profesorado inmerso en el proceso de enseñanza y aprendizaje de estos cursos académicos pudiese participar en la investigación, nos llevó a seleccionar una muestra representativa de los cuatro profesores-tutores y setenta y un alumnos de quinto y sexto de Educación Primaria.

\section{RESULTADOS}

Teniendo en cuenta todas las pruebas realizadas como son las tablas de frecuencias, los gráficos y las medidas de tendencia central nos dejan una idea clara de la veracidad de los objetivos.

Por ello, intentando reducir a lo más concreto y cierto los resultados de este trabajo, expondremos a continuación la verificación de los objetivos planteados, obteniendo las siguientes conclusiones:

1. Los agentes efectivos del proceso de enseñanza-aprendizaje, profesor y alumno, saben qué es una Pizarra Digital Interactiva.

2. Las aulas del Tercer Ciclo de Educación Primaria están dotadas de una Pizarra Digital Interactiva aunque no en todos los casos en las mejores condiciones de uso.

3. La mayoría del profesorado del Tercer Ciclo de Educación Primaria utiliza la Pizarra Digital Interactiva en alguna de sus clases.

4. El área de Conocimiento del Medio es la materia en la que más se utiliza la Pizarra Digital Interactiva.

5. El alumnado del Tercer Ciclo de Educación Primaria maneja en pocas ocasiones la Pizarra Digital Interactiva.

6. La utilización de la PDI en el aula fomenta la comprensión de los contenidos complejos.

7. La utilización de la PDI en el aula aumenta el interés por las clases.

8. La utilización de la PDI en el aula favorece la variedad de actividades.

9. La utilización de la PDI en el aula anima a participar en las actividades propuestas.

En las dos páginas siguientes se pueden observar, a modo de ejemplos, los gráficos correspondientes al análisis de resultados de las cuatro últimas conclusiones resultantes. 


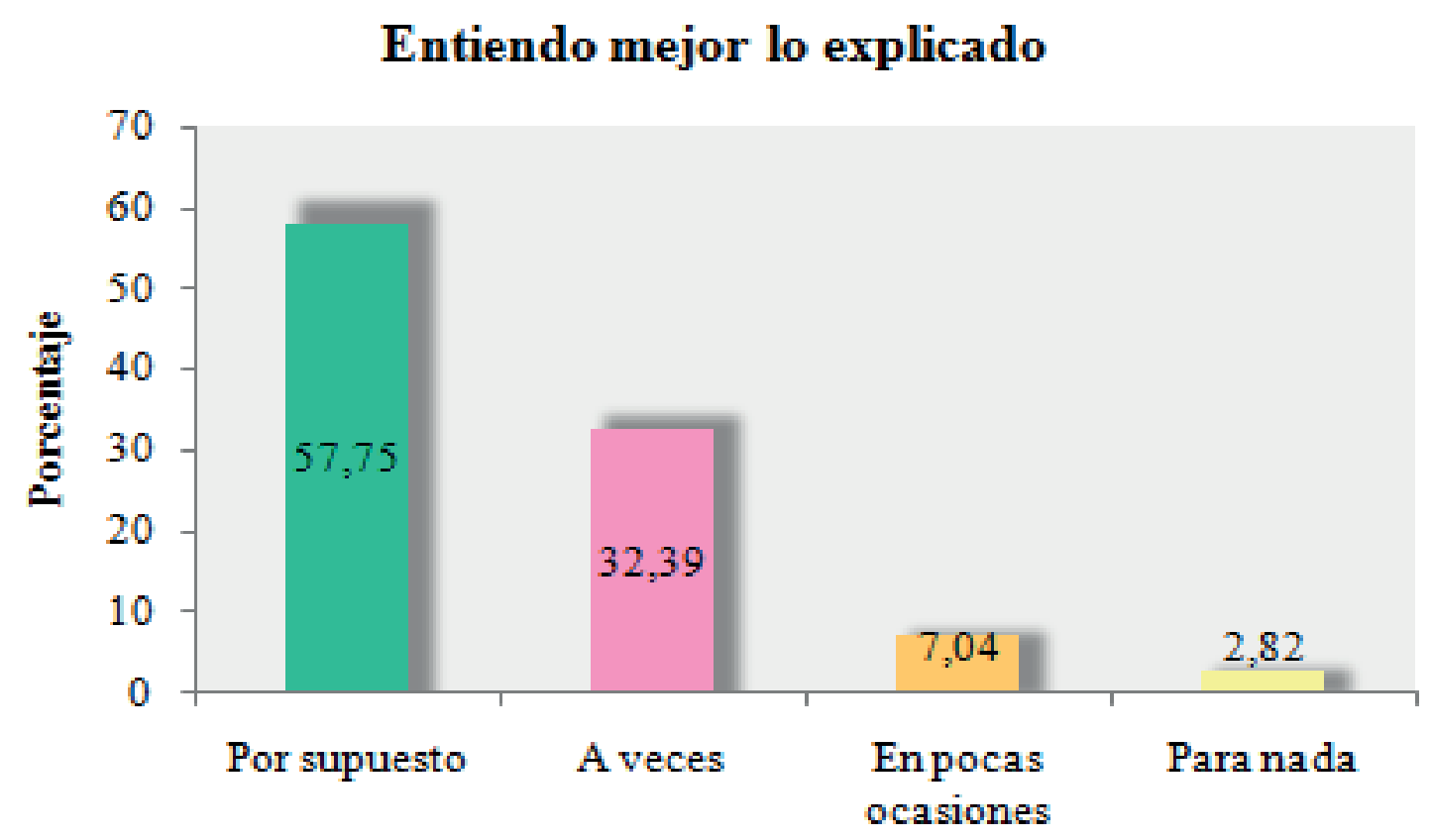

Gráfico 1: Entiendo mejor lo explicado.

Fuente: Propia.

\section{Me gustan más las clases}

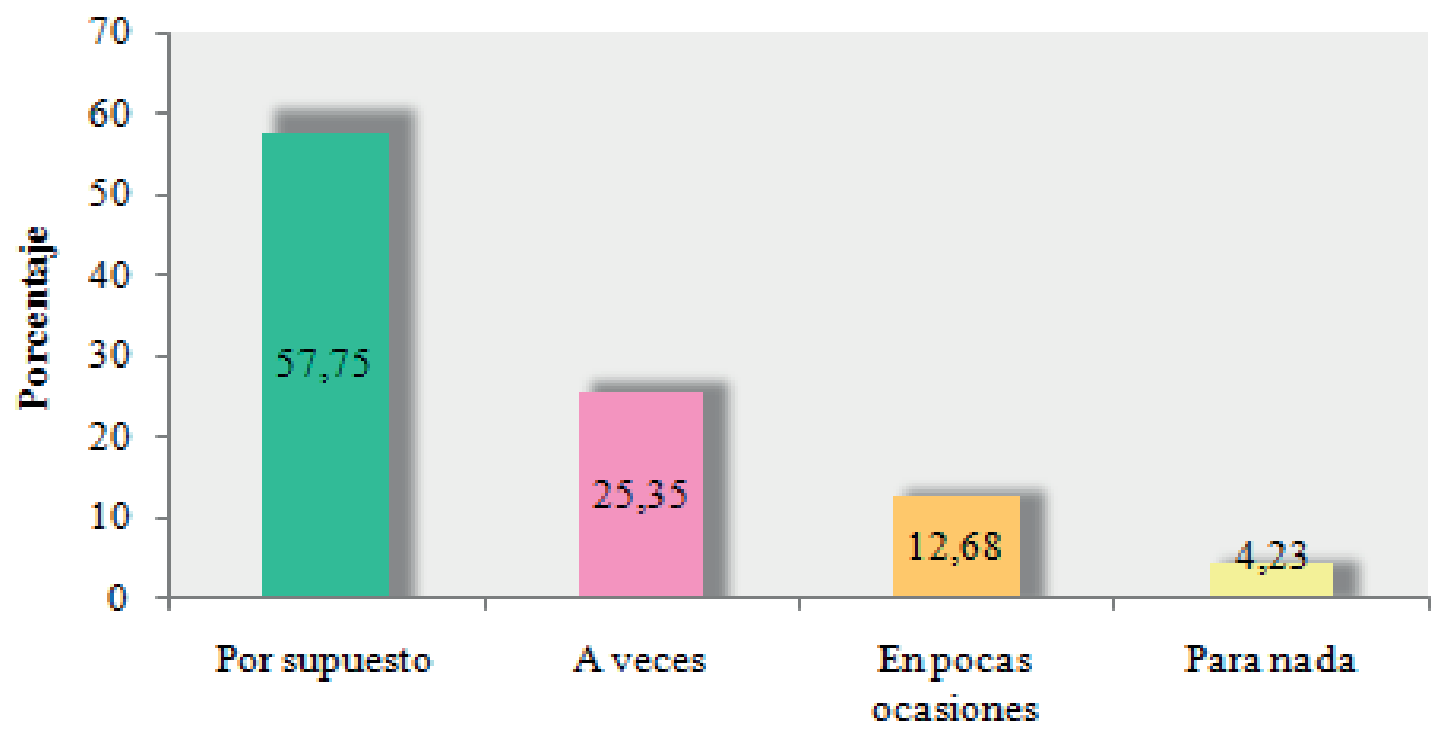

Gráfico 2: Me gustan más las clases.

Fuente: Propia. 


\section{Realizo una gran variedad de actividades}

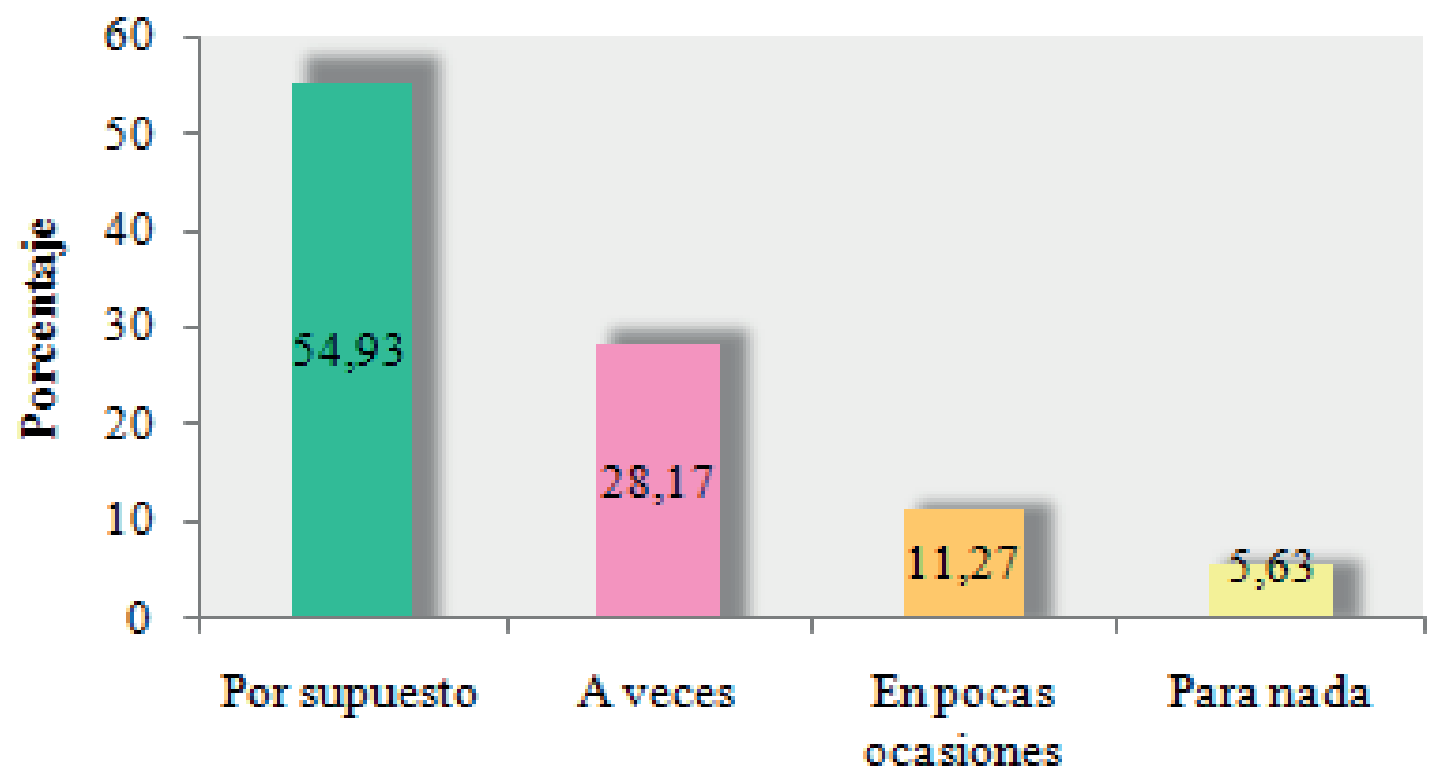

Gráfico 3: Realizo una gran variedad de actividades.

Fuente: Propia

\section{Participo más en las actividades}

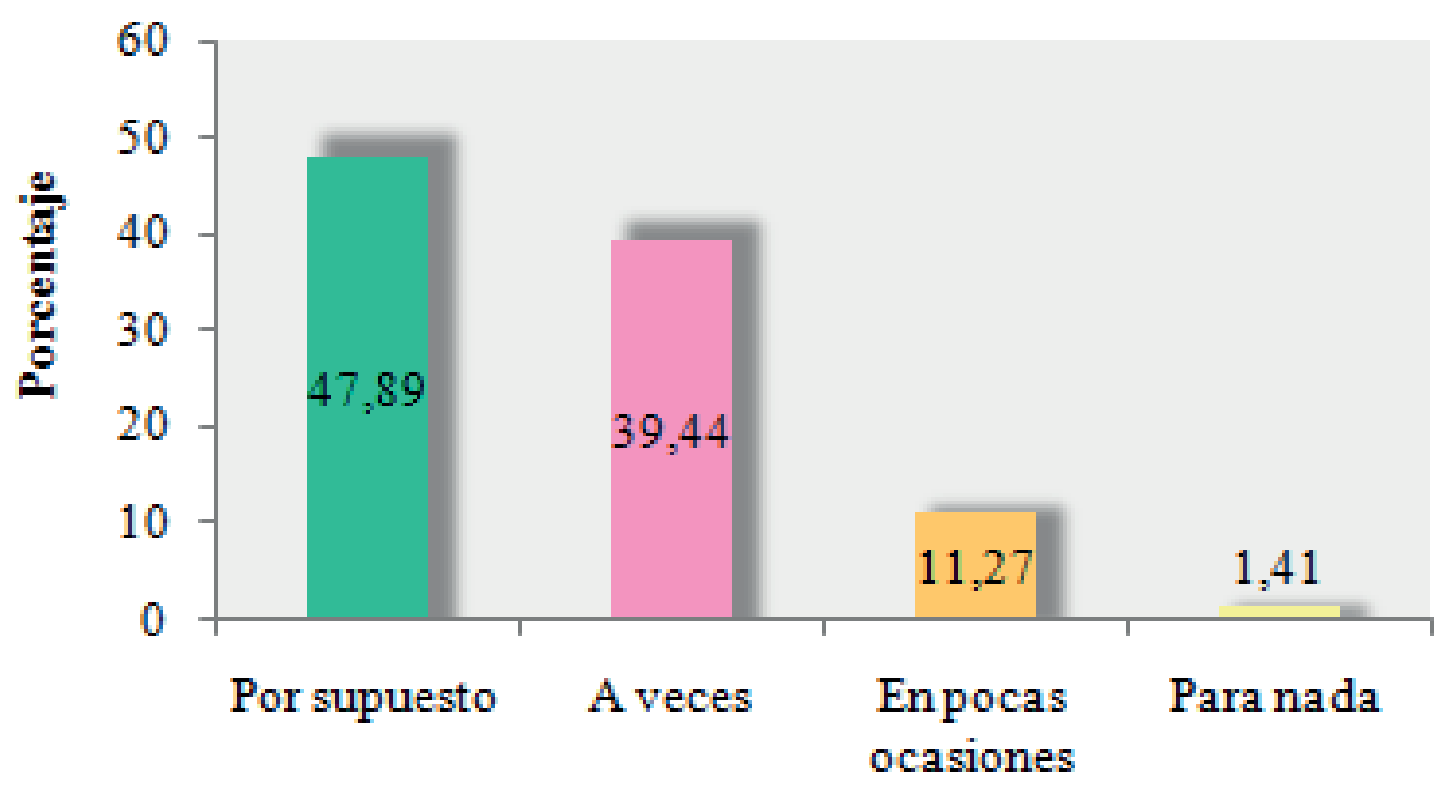

Gráfico 4: Participo más en las actividades.

Fuente: Propia 


\section{DISCUSIÓN. CONCLUSIONES.}

La obvia conclusión general que nos permite exponer todo lo visto hasta ahora, tanto en lo que a teoría se refiere como al estudio estadístico que se ha realizado, es la siguiente: la utilización de la Pizarra Digital Interactiva en el aula actúa como recurso potenciador de la motivación en los alumnos.

Con esto, queda probada la veracidad del título del presente trabajo dando por alcanzado los objetivos generales planteados.

No obstante, para finalizar este apartado hemos considerado oportuno realizar un análisis DAFO de la Pizarra Digital Interactiva en el caso investigado:

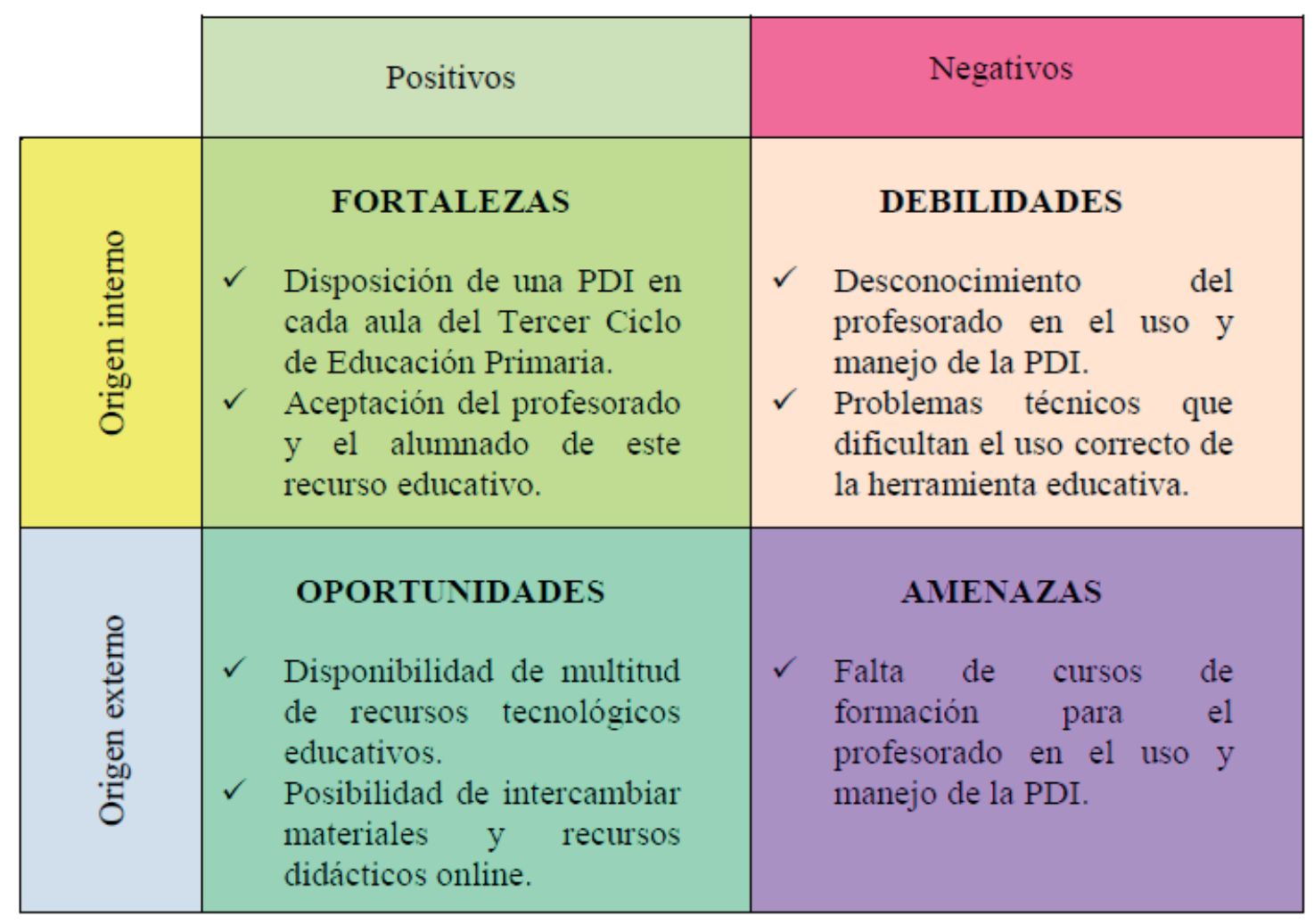

Gráfico 5: Análisis DAFO de la Pizarra Digital Interactiva. Fuente: Propia

Con esta técnica pretendemos mostrar la situación del centro educativo investigado en la integración de la Pizarra Digital Interactiva a su tarea docente, de modo que dicho diagnóstico sirva de estímulo para proponer actuaciones innovadoras en estas aulas, haciendo uso de esta herramienta educativa.

Por último y para finalizar este trabajo exponer que lo único que pretendemos es que sea un incentivo para otros futuros trabajos y artículos científicos de educación y al mismo tiempo resaltar la importancia y conveniencia de la utilización de las Tecnologías de la Información y la Comunicación en Educación, señalar la posibilidad de utilizar con éxito la Pizarra Digital Interactiva en las aulas y destacar la importancia de la motivación en los procesos de enseñanza-aprendizaje. 


\section{REFERENCIAS}

\section{Publicaciones Periódicas:}

Area, M. (2010). El proceso de integración y uso pedagógico de las TIC en los centros educativos. Estudio de casos. Recuperado de http://www.revistaeducacion.educacion.es/re352/re352_04.pdf

Armstrong, V., Barnes, S., Sutherland, R., Curran, S., Mills, S., y Thompson, I. (2005). Collaborative research methodology for investigating teaching and learning: the use of interactive whiteboard technology. Educational Review, 57(4), 455-467.

Beeland W. (2002). Student Engagement, Visual Learning and Technology: Can Interactive Whiteboards Help? Recuperado de http://chiron.valdosta.edu/are/Artmanscrpt/vol1no1/beeland_am.pdf

Bell, M. A. (2002). Why Use an Interactive Whiteboard? A Baker's dozen reasons! Recuperado de http://teachers.net/gazette/JAN02/mabell.html

Bryant, S. M., y Hunton, J. E. (2000). The use of technology in the delivery of instruction: Implications for accounting educators and education researchers. Issues in Accounting Education, 15(1), 129-162.

Cogill, J. (2002). How is the interactive whiteboard being used in the primary school and how does it affect teachers and teaching? Recuperado de http://www.virtuallearning.org.uk/whiteboards/IFS_Interactive_whiteboards _in_the_primary_school.pdf

Edwards J. A., Hartnell, M. y Martin, R. (2002). Interactive whiteboards: Some lessons from the classroom. Micromath, 18(2), 30-33.

Forrest, A. (2005). An interactive whiteboard in my classroom. Micromath, 21(3), 8.

Gage, J. (2002). So what is an electronic whiteboard? Should you want one? Micromath, 18(2), 5-7.

Gallego, D. J. y Gatica, N. (2000). La pizarra digital: una ventana al mundo desde las aulas. Sevilla: Eduforma.

Goodison, T. (2002). Learning with ICT at primary level: pupils'perceptions. Journal of Computer Assisted Learning, 18 (3), 282- 295. 
Hall, I., y Higgins, S. (2005). Primary school students' perceptions of interactive whiteboards. Journal of Computer assisted learning, 21(2), 102- 117.

Hodge, S., y Anderson B. (2007). Teaching and learning with an interactive whiteboard: a teacher's journey. Learning, Media and Technology, 32(3), 271282.

Holmes, K. (2009). Planning to teach with digital tools: Introducing the interactive whiteboard to pre-service secondary mathematics teachers. Australasian Journal of Educational Technology, 25(3), 351-365.

Kennewell, S. (2001): Interactive whiteboards - yet another solution looking for a problema to solve? Information Technology in Teacher Education, 30: Autumm 2001 Newsletter 39, 3-6.

Kennewell, S. and Morgan, A. (2003): Student teachers' experiences and attitudes towards using interactive whiteboards in the teaching and learning of young children. Proceedings of Young Children and Learning Technologies Conference. Sydney: IFIP.

Lee, M., y Boyle, M. (2003). The Educational Effects and Implications of the InteractiveWhiteboard Strategy of Richardson Primary School: a Brief Review. Recuperado

de http://www.richardsonps.act.edu.au/RichardsonReview_Grey.pdf

León, T. (2009). Dentro de poco tiempo las pizarras tradicionales no se verán en clase. Recuperado de

http:/ / www.diariodirecto.com/entrevistas/2009/11/12/proyecto-aula-digital163540065288.html

Levy, P. (2002). Interactive whiteboards in learning and teaching in two Sheffield Schools: a developmental study. Sheffield: Departament of Information Studies, University of Sheffield.

Marquès, P. y Grupo DIM. (2006). La pizarra digital en el aula de clase. Barcelona: Grupo Edebé.

Marqués, P. (2008). La pizarra digital. Recuperado de http://www.peremarques.net/pdigital/es/pizinteractiva.htm

McClelland, D. (1955). Studies in Motivation. Nueva York: Appleton-CenturyCrofts. 
Miller D. y Glover D. (2002). The Interactive Whiteboard as a Force for Pedagogic Change: The Experience of Five Elementary Schools in an English Education Authority. Recuperado de http:/ / www.aace.org/files/ITCE2002215.pdf

Miller, D., y Glover, D. (2006). Interactive whiteboard evaluation for the secondary national strategy. Developing the use of Interactive Whiteboards in Mathematics. Recuperado de http://www.standards.dfes.gov.uk/secondary/keystage3/downloads/ma_ia w_eval_rpt.pdf

Pugh, M. (2001). Using an Interactive Whiteboard with SLD Students. Consultada el 7 de marzo de 2013, en http:/ / ferl.becta.org.uk/display.cfm?resID=1393

Puig, V. (2006). Más confusión en las aulas. Recuperado de http://www.elnortedecastilla.es/pg060307/prensa/noticias/Articulos_Opinio n/200603/07/VAL-OPI-243.html

Quashie, V. (2009). How interactive is the interactive whiteboard? Mathematics Teaching, 214, 33-38.

RED.es. (2006). La Pizarra Interactiva como recurso interactivo en el aula. Recuperado de http://web.educastur.princast.es/proyectos/cuate/docum/pdi_redes.pdf

San Pedro, J. C. (2008). Pizarras digitales interactivas: potencialidad y características. La irrupción en las aulas en la web 2.0. En M. Del Moral y R. Rodríguez (Coords.), Experiencias docentes y TIC, 229-248.

SMART Technologies Inc. (2005). Pizarras digitales interactivas y aprendizaje: una revisión de estudios de casos e investigaciones. Recuperado de http://reddigital.cnice.mec.es/6/Documentos/docs/otrosdoc04_material.pdf

Smith, A. (2000). Interactive Whiteboard Evaluation. Recuperado de http:/ / www.mirandanet.ac.uk/pubs/smartboard.htm

Smith, H., Higgins, S., Wall, K. y Miller, J. (2005). Interactive whiteboards: boon or bandwagon? A critical review of the literature. Journal of Computer Assisted Learning 21, 91-101.

\section{Capítulo de un libro o entrada de un libro de consulta:}

Bustos, A. (2001). ¿Cómo evitar el fracaso escolar?: estrategias de solución. Barcelona: Erasmus Ediciones. 
Clemens, A., Moore, T. y Nelson, B. (2001). Math intervention "SMART" project (student mathematical analysis and reasoning with technology). Recuperado de http://smarterkids.org/research/paper10.asp.

Gallego, D. y Dulac, J. (2005). Informe final Iberian Research Project. Recuperado de http:/ / www.dulac.es/Iberian\%20research/IberianResearchProject.htm

\section{Libros completes}

Madsen, K.B. (1972). Teorías de la motivación. Buenos Aires: Paidós.

McDougall, W. (1932). The Energies of Men. London: Methuen.

Tolman, E.C. (1932). Purposive Behavior in Animals and Men. New York: Century.

\section{AUTORES:}

\section{Celia González Carrasco:}

Egresada en Magisterio con la especialidad de Primaria en la Facultad de Educación de Toledo.

\section{José Francisco Durán Medina:}

Profesor Doctor en la Universidad de Castilla La Mancha. Facultad de Educación. Departamento de Pedagogía.

Investigación: Participación en numerosos Proyectos de Investigación Nacionales, como Socmedia (UCM Madrid), Competencia en Comunicación Audiovisual (UPF Barcelona), Ciberimaginario (UJRC Madrid), Un Ordenador por Niño (La Laguna Canarias); igualmente participación en Proyectos de Investigación Internacionales, como MIMETIC (Poitiers Francia).

Publicaciones: Numerosos capítulos de libros dedicados la incorporación de TIC en las aulas. Del mismo modo, abundantes artículos en revistas digitales indexadas e impresas.

Congresos: gran cantidad de ponencias y comunicaciones en diferentes congresos internacionales y de variadas ciudades españolas, como Sevilla, Barcelona, Cuenca, Madrid, Toledo...

ORCID: 0000-0002-9444-4029

REDIRIS: JoseFrancisco.Duran@uclm.es: EDUTEC-L, EDUTIC.

RESEARCH GATE: $\underline{\text { https://www.researchgate.net/profile/Jose_Duran_Medina }}$ 